\title{
Different latencies of edge detection cause the fluttering-heart illusion
}

\author{
Masahiro Suzuki ${ }^{1} \cdot$ Kazuhisa Yanaka $^{1}$
}

Received: 7 June 2020 / Accepted: 20 January 2021 / Published online: 16 February 2021

(c) The Author(s) 2021

\begin{abstract}
We studied the mechanism causing the fluttering-heart illusion in which the motion of an inner figure appears unsynchronized compared with that of the outer figure surrounding it although the motion of both figures is objectively synchronized in reality. Experiment 1 examined the effect of edges' luminance contrasts. The illusion was measured under conditions where the luminance contrasts of the outer and inner figures' edges were varied. The results indicated that the illusion occurred when the outer figure's edge had a high luminance contrast and the inner figure's edge had a low luminance contrast and that the illusion was reversed when the outer figure's edge had a low luminance contrast and the inner figure's edge had a high luminance contrast. Experiment 2 examined the effect of the first- and second-order edges. The illusion was measured under conditions where the first- and second-order edges coexisted or only the first-order edges existed. The results indicated that the illusion occurred when the outer figure had the first-order edge and the inner figure had the second-order edge, and that the illusion was reversed when the outer figure had the second-order edge and the inner figure had the first-order edge. These findings supported the hypothesis that the different latencies of edge detection cause the fluttering-heart illusion.
\end{abstract}

Keywords Fluttering-heart illusion · Optical illusion · Edge detection $\cdot$ Luminance contrast $\cdot$ First-order edge $\cdot$ Secondorder edge

\section{Introduction}

The fluttering-heart illusion is a phenomenon where the movements of inner figures appear unsynchronized with those outer figures surrounding them although the movements of both figures are objectively synchronized in reality [1-3]. Figure 1 shows a typical stimulus of the flutteringheart illusion; the background is a piece of white paper, the outer figure is a blue disk, and the inner figure is red and heart-shaped. When an observer moves the paper with his/ her hand, the outer blue disk's motion appears synchronized with the hand movement; however, in comparison, the inner red heart-shaped figure's motion appears delayed. In this study, we examined the mechanism causing the flutteringheart illusion.

Masahiro Suzuki

msuzuki@ctr.kanagawa-it.ac.jp

Kazuhisa Yanaka

yanaka@ic.kanagawa-it.ac.jp

1 Kanagawa Institute of Technology, 1030 Shimo-ogino, Atsugi, Kanagawa 243-0292, Japan
Most previous studies have argued that the mechanism causing the fluttering-heart illusion is related to color information processing. Initial studies argued that the latencies of color information processing differ between colors and that these differences cause the fluttering-heart illusion [1-3]. Some studies indicated that the different latencies between colors that cause the fluttering-heart illusion are related to rod and cone cell characteristics [4-8]. Other studies showed that motion defined by color is perceived more slowly compared to motion defined by luminance, arguing that the fluttering-heart illusion can be explained based on differently perceived velocities between inner and outer figures [9]. Thus, the fluttering-heart illusion has been considered a phenomenon that occurs based on color information processing.

However, other previous studies reported phenomena that cannot be explained based on color information processing [10]. In the phenomena, the movements of monochrome figures with a texture whose luminance contrast is low appear delayed against the movements of monochrome figures with a texture whose luminance contrast is high. Although such phenomena can be explained based on the fast latency of texture with high luminance contrast and the slow latency of texture with low luminance contrast, this explanation does 


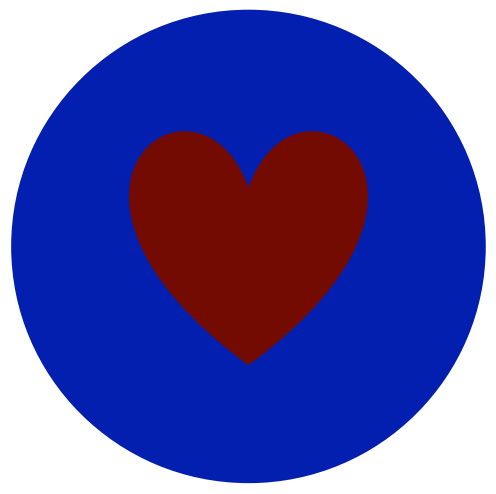

Fig. 1 Typical stimulus of the fluttering-heart illusion

not meet the phenomenon that can be explained based on color information processing, e.g., the fluttering-heart illusion that occurs when presented with a typical stimulus (see Fig. 1).

Extending these previous studies, we propose a new hypothesis to explain a series of phenomena. This hypothesis focuses on the edges of outer and inner figures. For example, in a typical stimulus (see Fig. 1), the outer figure's edge comprises a white background which is a piece of paper and the blue disk which is painted on the paper. On the other hand, the inner figure's edge comprises the blue disk and the red heart-shaped figure which are painted on the paper. That is, the luminance contrast of the outer figure's edge is high and that of the inner figure's edge is low. According to previous studies, the latencies of detection of edges with low luminance contrasts are slow, and the latencies of detection of edges with high luminance contrasts are fast [11-17]. Therefore, we can hypothesize that the differing latencies of edge detection between the outer and inner figures cause the fluttering-heart illusion even when a typical stimulus is observed (Fig. 2a, b). Moreover, we can hypothesize that the fluttering-heart illusion occurs because of differing latencies of texture detection between the outer and inner figures when their edges have the same luminance contrasts. Thus, a series of phenomena can be explained based on this hypothesis. We call it the edge detection hypothesis.

To examine the edge detection hypothesis, we use the working hypothesis that edges' luminance contrast determines the direction and amount of the fluttering-heart illusion (Fig. 2a-d). That is, differences in edge detection latencies between the outer and inner figures cause the fluttering-heart illusion even when an achromatic stimulus is observed. The movements of figures whose edges have low luminance contrasts appear delayed against the movements of figures whose edges have high luminance contrasts. The amount of the fluttering-heart illusion decreases when the luminance contrasts of the outer and inner figures' edges are similar. We can evaluate this working hypothesis by examining the effect of the edges' luminance contrasts on the fluttering-heart illusion.

We also use another working hypothesis, namely, the fluttering-heart illusion occurs when first- and second-order edges coexist (Fig. 2e, f). According to previous studies, second-order edges are detected by using a higher process than the detection of first-order edges [18-23], which indicates that the latencies of detection of second-order edges are slower than the latencies of detection of first-order edges. Therefore, differences in the latencies of detection of firstand second-order edges cause the fluttering-heart illusion. We can evaluate this working hypothesis by examining the effect of first- and second-order edges on the fluttering-heart illusion.

Therefore, this paper describes two experiments. In Experiment 1, the effect of edges' luminance contrasts on the fluttering-heart illusion is examined to evaluate the first working hypothesis. In Experiment 2, the effect of first- and second-order edges on the fluttering-heart illusion is examined to evaluate the second working hypothesis. Thus, the edge detection hypothesis is evaluated by examining these two working hypotheses.

\section{Experiment 1: effect of edges' luminance contrasts}

\subsection{Purpose}

We examine the effect of edges' luminance contrasts on the fluttering-heart illusion to evaluate the first working hypothesis: edges' luminance contrasts determine the direction and amount of the fluttering-heart illusion. In this experiment, the stimuli's background has a high luminance, inner figures have a low luminance, and outer figures have some conditions of luminance (Fig. 3). Under conditions in which the outer figure's luminance is close to the background but far from the inner figure, the outer figure's edges have a low luminance contrast and the inner figure's edges have a high luminance contrast (Fig. 3a, c). Under conditions in which the outer figure's luminance is far from the background but close to the inner figure, the outer figure's edges have a high luminance contrast and the inner figure's edges have a low luminance contrast (Fig. 3b, d). We measure the direction and amount of the fluttering-heart illusion under these conditions.

We also have two conditions of color in stimuli (Fig. 3). Under the chromatic condition, the background is white, the outer figure is blue, and the inner figure is red (Fig. 3a, b). This coloring is the same as the typical stimulus presented in Fig. 1. Under the achromatic condition, the background is white, the outer figure is gray, and the inner figure is black (Fig. 3c, d). To examine the effect of the luminance contrasts 
Fig. 2 Illustration showing how different latencies of edge detection cause the fluttering-heart illusion. a The outer figure's edge has a high luminance contrast, and the inner figure's edge has a low luminance contrast; therefore, the inner figure has a slower latency of edge detection than the outer figure. b When the outer and inner figures move, the inner figure's perceived position is delayed against its actual position due to a slower latency, although the outer figure's perceived position is not delayed. $\mathbf{c}$ The outer figure's edge has a low luminance contrast, and the inner figure's edge has a high luminance contrast; therefore, the outer figure has a slower latency of edge detection than the inner figure. d When the outer and inner figures move, the outer figure's perceived position is delayed against its actual position due to a slower latency, although the inner figure's perceived position is not. e The outer figure's edge is defined by luminance, i.e., the first-order edge, and the inner figure is given a temporally modulated luminance that the mean luminance is the same as the outer figure's luminance, i.e. the inner figure's edge is the second-order edge. $\mathbf{f}$ When the outer and inner figures move, the inner figure's perceived position is delayed against its actual position, although the outer figure's perceived position is not

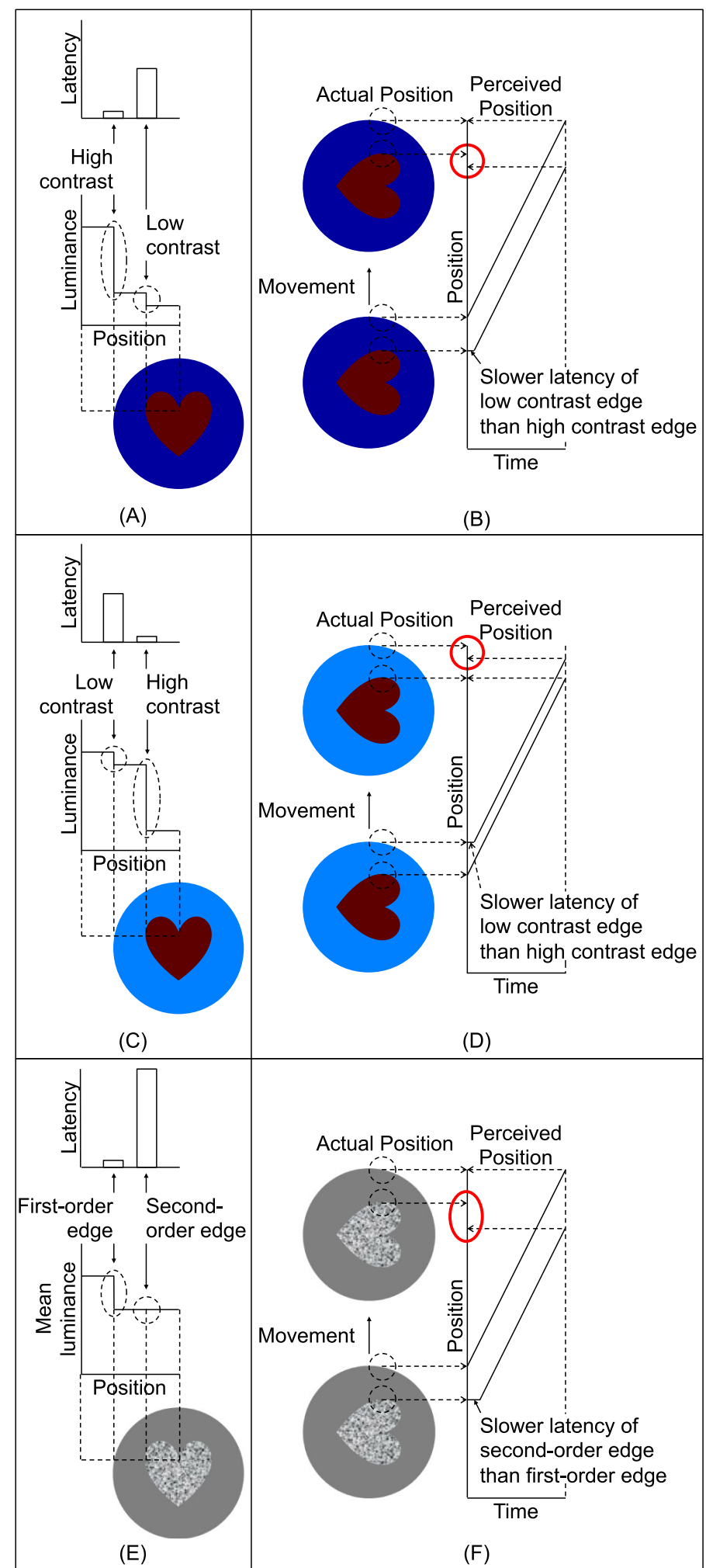

\subsection{Method}

\subsubsection{Apparatus}

The experiment was conducted in a dark room. Stimuli 


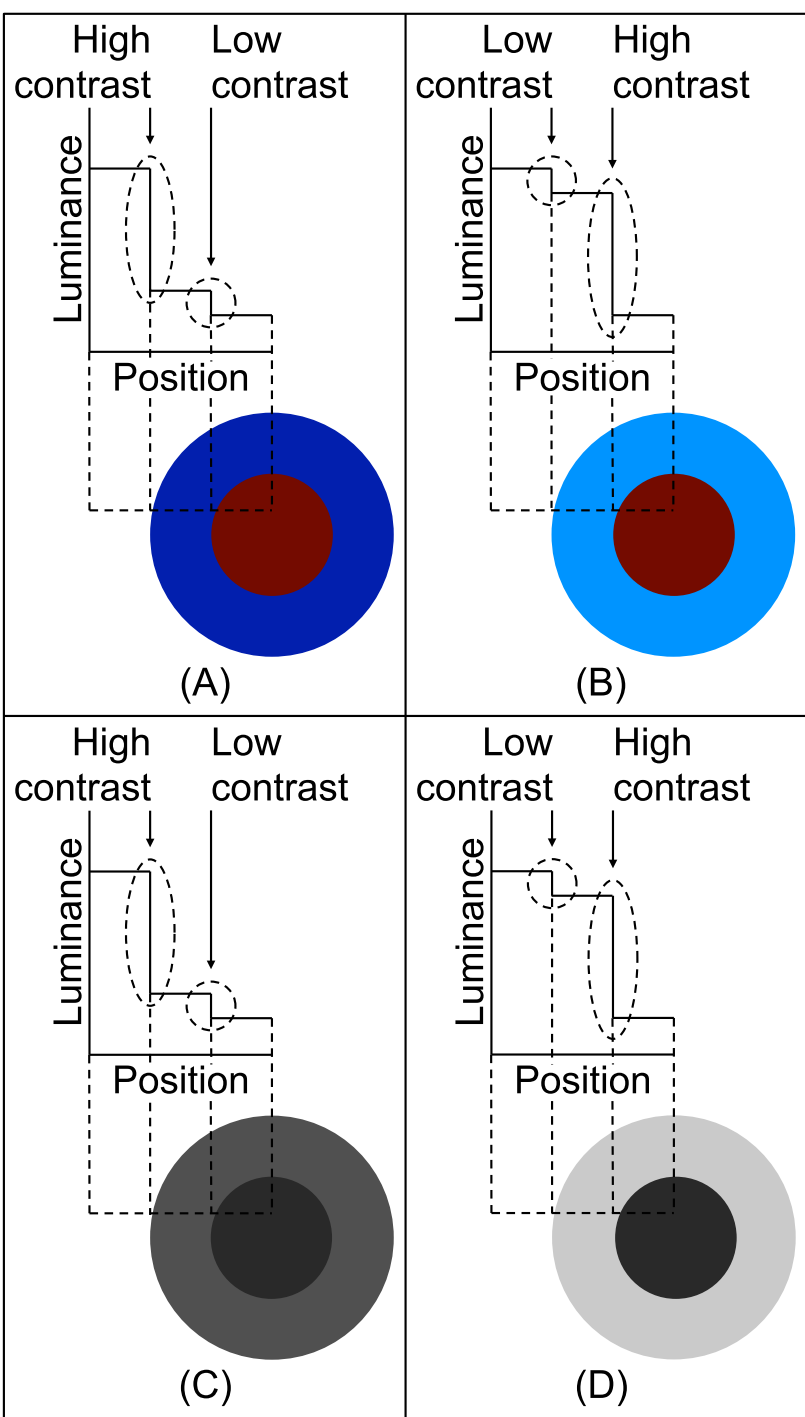

Fig. 3 Schematic of stimuli used in Experiment 1. a Chromatic condition with the outer disk's low luminance. b Chromatic condition with the outer disk's high luminance. c Achromatic condition with the outer disk's low luminance. d Achromatic condition with the outer disk's high luminance

were generated with a workstation (Precision 390, Dell, Round Rock, TX) and a 23-inch liquid-crystal display (RDT233WX-3D, Mitsubishi Electric, Tokyo, Japan). The refresh rate of the workstation's video card was $60 \mathrm{~Hz}$. The display was positioned in front of an observer whose head was secured with a chin rest and a forehead rest. The viewing distance was $57.3 \mathrm{~cm}$. The observer's response was input into the workstation using a numerical keypad in the observer's hand.

\subsubsection{Stimuli}

Figure 3 shows the schematic of the stimuli used in Experiment 1 . The stimuli comprised a background, an outer disk, and an inner disk. The background was a luminance of $10.0 \mathrm{~cd} / \mathrm{m}^{2}$, the outer disk was $0.50,3.00,5.50$, or $8.00 \mathrm{~cd} /$ $\mathrm{m}^{2}$, and the inner disk was $0.30 \mathrm{~cd} / \mathrm{m}^{2}$. Under the chromatic condition, the background was 0.33 of $x$ and 0.33 of $y$ in the International Commission on Illumination (CIE) $x y Y$ color space; the outer disk was 0.14 of $x$ and 0.07 of $y$; and the inner disk was 0.50 of $x$ and 0.30 of $y$. Under the achromatic condition, the background, outer disk, and inner disk were 0.33 of $x$ and 0.33 of $y$. The outer disk's diameter was $10.0^{\circ}$, and the inner disk's diameter was $5.00^{\circ}$. The centers of the disks were $0.50^{\circ}$ from the screen's center, and the disks were moved clockwise in keeping with this eccentricity. The velocity of the disks' movements was $4 \pi, 6 \pi$, $8 \pi$, or $10 \pi \mathrm{rad} / \mathrm{s}$. The phase difference between the disks' movements was changed by keypad inputs. The step size of changing the phase difference was $\pi / 180 \mathrm{rad}$. No fixation point was presented.

\subsubsection{Observers}

Five employees and one undergraduate student from the Kanagawa Institute of Technology participated in Experiment 1 . Two of them were the authors and the rest were naive to the purpose of the experiment. All observers had normal or corrected-to-normal visual acuity and normal color vision.

\subsubsection{Procedure}

Before beginning the trials, candidates for observers were asked to orally report their experience of the test for visual acuity or color vision deficiency. The candidates who reported that they had normal or corrected-to-normal visual acuity and normal color vision were then asked to participate in the experiment. Informed consent was obtained for experimentation with human subjects. The work was conducted in accordance with the Code of Ethics of the World Medical Association (Declaration of Helsinki).

Observers were asked to gaze at the moving disks and to adjust the phase difference until they observed the inner and outer disks' movements as synchronized. For each trial, the experimenter randomly adjusted the phase difference until the disks' movements were clearly unsynchronized, and then the observers initiated their adjustments. When pressing the " 4 " and " 6 " keys of the keypad, the phase difference was changed by shifting the inner disk's phase counterclockwise and clockwise, respectively. The observers were informed which key should be pressed first to decrease the disks' unsynchronized movements at the start of each trial. During 
this trial, the observers pressed the keys to decrease the disks' unsynchronized movements and, when they observed the disks' synchronized movements, they pressed the "enter" key of the keypad to complete the trial. For each trial, the final phase difference was recorded as the point of subjective equality (PSE). Each observer performed ten trials for each condition.
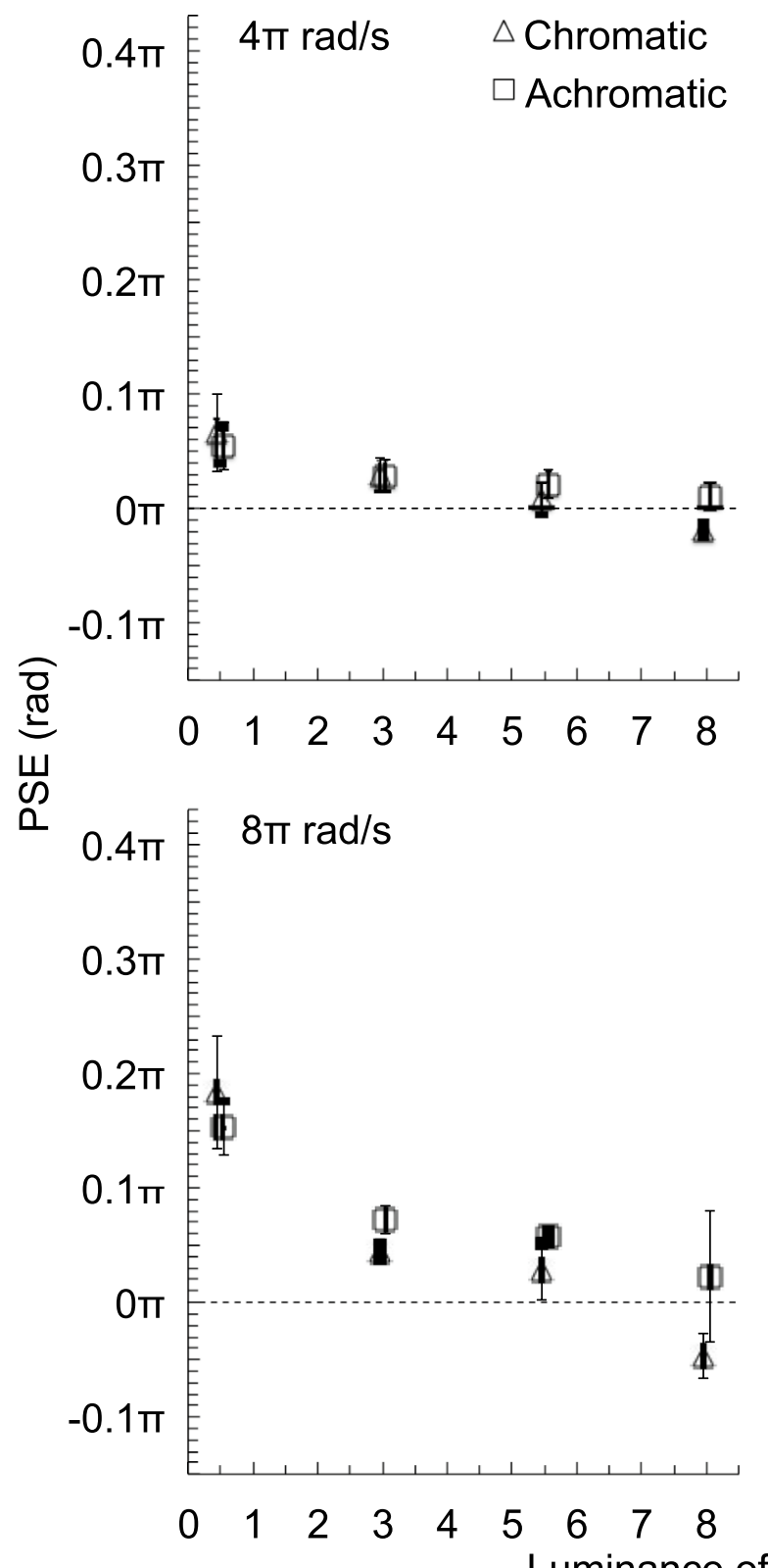

\subsection{Results}

Each observer's mean PSE was first calculated based on 10 trials for each condition, and the six observers' mean PSE and $95 \%$ confidence interval were then calculated according to each observer's mean PSE for each condition. Figure 4 shows the six observers' mean PSE plus/minus 95\%
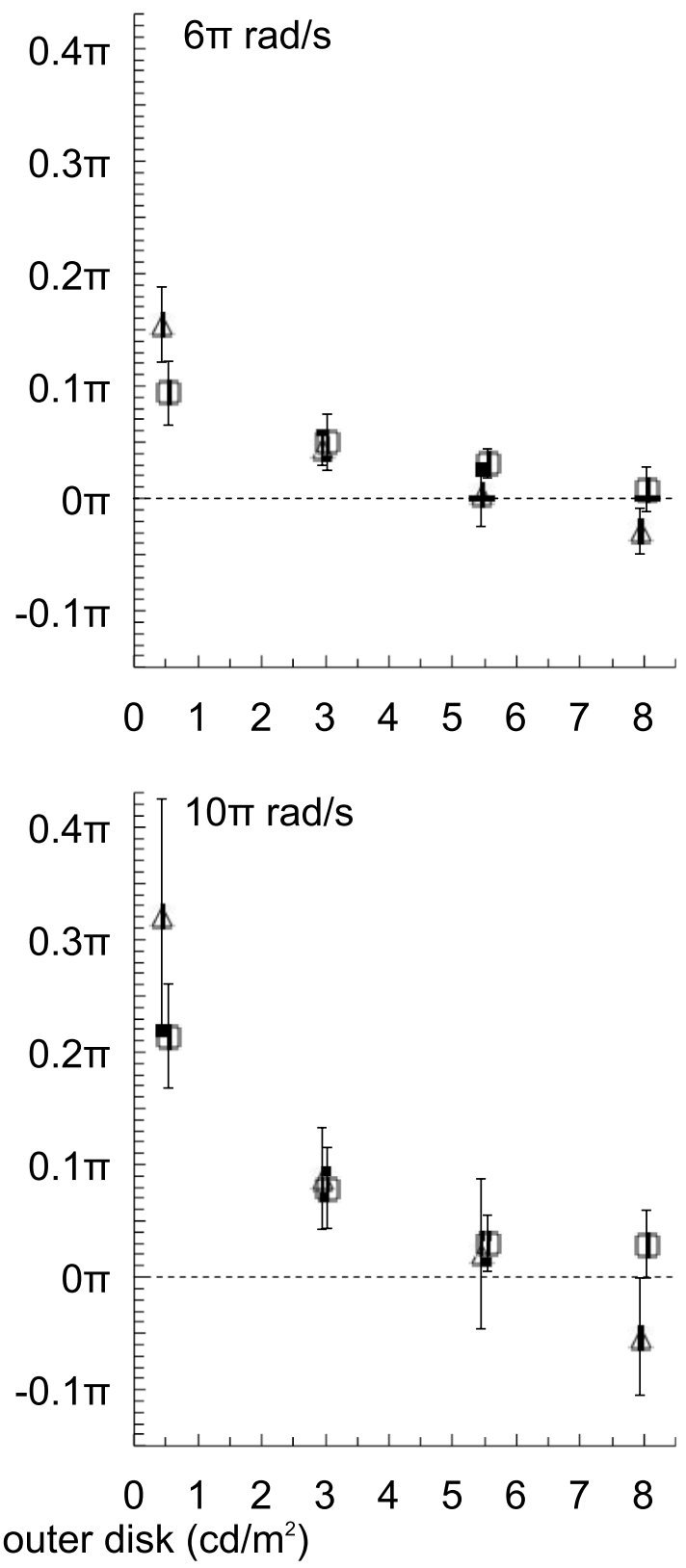

Fig. 4 Six observers' mean PSE plus/minus 95\% confidence interval as a function of the outer disk's luminance. The horizontal axis is the outer disk's luminance, and the vertical axis is PSE. A positive PSE indicates that the phase of the inner disk's movement differs from that of the outer disk's movement in the same direction as the disks' movements. That is, the inner disk's movement appears delayed against the outer disk's movement. A negative PSE indicates that the phase of the inner disk's movement differs from that of the outer disk's movement in the opposite direction to the disks' movements. That is, the outer disk's movement appears delayed against the inner disk's movement. The triangles and squares represent results under chromatic and achromatic conditions, respectively. The error bar represents the $95 \%$ confidence interval 
confidence interval as a function of the outer disks' luminance. The 95\% confidence interval did not include $0 \pi \mathrm{rad}$ of PSE under the chromatic conditions of 0.50 and $3.00 \mathrm{~cd} /$ $\mathrm{m}^{2}$ of all the velocity conditions, and the PSE had a positive value. The $95 \%$ confidence interval also did not include $0 \pi$ rad of PSE under the achromatic conditions of 0.50, 3.00, and $5.50 \mathrm{~cd} / \mathrm{m}^{2}$ of all the velocity conditions, and the PSE had a positive value. These results indicate that the inner disk's movement appears delayed against the outer disk's movement. The $95 \%$ confidence interval did not include $0 \pi$ $\mathrm{rad}$ of PSE under the chromatic conditions of $8.00 \mathrm{~cd} / \mathrm{m}^{2}$ of all the velocity conditions, but the PSE had a negative value. These results indicate that the outer disk's movement appears delayed against the inner disk's movement. Thus, the fluttering-heart illusion occurs under these conditions, but the direction of illusion is reversed under the chromatic conditions of $8.00 \mathrm{~cd} / \mathrm{m}^{2}$ of all the velocity conditions.

A three-way (outer disk's luminance $\times$ color $\times$ velocity of disks' movements) analysis of variance (ANOVA) was conducted using Huynh-Feldt $\varepsilon$ because Mauchly's sphericity test rejected the sphericity assumption. Tables 1 and 2 show the results of those statistical tests. The second-order interaction was insignificant. The interactions between the outer disk's luminance and color, and between the outer disk's luminance and the velocity of the disks' movements were significant. The interaction between the color and the velocity of the disks' movements was insignificant. The effects of the outer disk's luminance and the velocity of the disks' movements were significant. The effect of color was insignificant. These results indicate that the effect of the outer disk's luminance interacts with color or with the velocity of the disks' movements.

Multiple comparisons using Bonferroni's method were conducted to further examine the interaction between the outer disk's luminance and color. Table 3 shows the results of those statistical tests. Under the chromatic condition, except for the pair in the $3.00-$ and $5.50-\mathrm{cd} / \mathrm{m}^{2}$ conditions and the pair in the $5.50-$ and $8.00-\mathrm{cd} / \mathrm{m}^{2}$ conditions, all pairs of the outer disk's luminance had significant differences. Under the achromatic condition, except for the pair in the 5.50- and $8.00-\mathrm{cd} / \mathrm{m}^{2}$ conditions, all pairs of the outer disk's luminance had significant differences. These results indicate that the luminance contrasts of the outer and inner disks' edges affect the fluttering-heart illusion

Table 1 Results of the Mauchly's sphericity test in Experiment 1

\begin{tabular}{lcccc}
\hline Factor & $W$ & $\chi^{2}$ & $d f$ & $P$ \\
\hline Outer disk's luminance & .056 & 10.7 & 5 & .066 \\
Velocity of disks' movements & .007 & 18.4 & 5 & .003 \\
Outer disk's luminance $\times$ Color & .076 & 9.60 & 5 & .097 \\
Color $\times$ Velocity of disks' movements & .083 & 9.26 & 5 & .109 \\
\hline
\end{tabular}

Table 2 Results of the three-way ANOVA in Experiment 1

\begin{tabular}{|c|c|c|c|c|c|}
\hline Factor & $F$ & $d f$ & $M S E$ & $p$ & $\eta_{\mathrm{p}}^{2}$ \\
\hline $\begin{array}{l}\text { Outer disk's lumi- } \\
\text { nance } \times \text { Color } \times \text { Velocity of } \\
\text { disks' movements }\end{array}$ & 2.56 & $4.97,24.8$ & 0.02 & .054 & .338 \\
\hline Outer disk's luminance $\times$ Color & 25.9 & $2.40,12.0$ & 0.01 & .000 & .838 \\
\hline $\begin{array}{l}\text { Outer disk's luminance } \times \text { Veloc- } \\
\text { ity of disks' movements }\end{array}$ & 24.6 & $7.09,35.5$ & 0.01 & .000 & .831 \\
\hline $\begin{array}{l}\text { Color } \times \text { Velocity of disks' move- } \\
\text { ments }\end{array}$ & 2.87 & $2.91,14.5$ & 0.01 & .074 & .365 \\
\hline Outer disk's luminance & 103 & $1.88,9.38$ & 0.04 & .000 & .954 \\
\hline Color & 0.86 & $1.00,5.00$ & 0.03 & .397 & .146 \\
\hline Velocity of disks' movements & 17.1 & $1.12,5.61$ & 0.06 & .006 & .774 \\
\hline
\end{tabular}

and such effects differ between chromatic and achromatic conditions.

Multiple comparisons using Bonferroni's method were also conducted to further examine the interaction between the outer disk's luminance and the velocity of the disks' movements. Table 4 shows the results of those statistical tests. Under the condition of $4 \pi \mathrm{rad} / \mathrm{s}$, except for the pair in the 3.00- and $8.00-\mathrm{cd} / \mathrm{m}^{2}$ conditions and the pair in the $5.50-$ and $8.00-\mathrm{cd} / \mathrm{m}^{2}$ conditions, all pairs of the outer disk's luminance had significant differences. Under the conditions of $6 \pi, 8 \pi$, and $10 \pi \mathrm{rad} / \mathrm{s}$, except for the pair in the 3.00- and $5.50-\mathrm{cd} / \mathrm{m}^{2}$ conditions and the pair in the $5.50-$ and $8.00-\mathrm{cd} / \mathrm{m}^{2}$ conditions, all pairs of the outer disk's luminance had significant differences. These results indicate that the luminance contrasts of the outer and inner disks' edges affect the fluttering-heart illusion and such effects depend on the velocity of the disks' movements.

Table 3 Results of the multiple comparisons to examine the interaction between the outer disk's luminance and color in Experiment 1

\begin{tabular}{lll}
\hline Color & Outer disk's luminance & Significance \\
\hline Chromatic & $0.50 \mathrm{~cd} / \mathrm{m}^{2}$ vs. $3.00 \mathrm{~cd} / \mathrm{m}^{2}$ & $p<.05$ \\
& $0.50 \mathrm{~cd} / \mathrm{m}^{2}$ vs. $5.50 \mathrm{~cd} / \mathrm{m}^{2}$ & $p<.05$ \\
& $0.50 \mathrm{~cd} / \mathrm{m}^{2}$ vs. $8.00 \mathrm{~cd} / \mathrm{m}^{2}$ & $p<.05$ \\
& $3.00 \mathrm{~cd} / \mathrm{m}^{2}$ vs. $5.50 \mathrm{~cd} / \mathrm{m}^{2}$ & $n . s$ \\
& $3.00 \mathrm{~cd} / \mathrm{m}^{2}$ vs. $8.00 \mathrm{~cd} / \mathrm{m}^{2}$ & $p<.05$ \\
& $5.50 \mathrm{~cd} / \mathrm{m}^{2}$ vs. $8.00 \mathrm{~cd} / \mathrm{m}^{2}$ & $n . s$ \\
Achromatic & $0.50 \mathrm{~cd} / \mathrm{m}^{2}$ vs. $3.00 \mathrm{~cd} / \mathrm{m}^{2}$ & $p<.05$ \\
& $0.50 \mathrm{~cd} / \mathrm{m}^{2}$ vs. $5.50 \mathrm{~cd} / \mathrm{m}^{2}$ & $p<.05$ \\
& $0.50 \mathrm{~cd} / \mathrm{m}^{2}$ vs. $8.00 \mathrm{~cd} / \mathrm{m}^{2}$ & $p<.05$ \\
& $3.00 \mathrm{~cd} / \mathrm{m}^{2}$ vs. $5.50 \mathrm{~cd} / \mathrm{m}^{2}$ & $p<.05$ \\
& $3.00 \mathrm{~cd} / \mathrm{m}^{2}$ vs. $8.00 \mathrm{~cd} / \mathrm{m}^{2}$ & $p<.05$ \\
& $5.50 \mathrm{~cd} / \mathrm{m}^{2}$ vs. $8.00 \mathrm{~cd} / \mathrm{m}^{2}$ & $n . s$ \\
\hline
\end{tabular}


Table 4 Results of the multiple comparisons to examine the interaction between the outer disk's luminance and the velocity of the disks' movements in Experiment 1

\begin{tabular}{lll}
\hline $\begin{array}{l}\text { Velocity of disks' } \\
\text { movements }\end{array}$ & Outer disk's luminance & Significance \\
\hline $4 \pi \mathrm{rad} / \mathrm{s}$ & $0.50 \mathrm{~cd} / \mathrm{m}^{2}$ vs. $3.00 \mathrm{~cd} / \mathrm{m}^{2}$ & $p<.05$ \\
& $0.50 \mathrm{~cd} / \mathrm{m}^{2}$ vs. $5.50 \mathrm{~cd} / \mathrm{m}^{2}$ & $p<.05$ \\
& $0.50 \mathrm{~cd} / \mathrm{m}^{2}$ vs. $8.00 \mathrm{~cd} / \mathrm{m}^{2}$ & $p<.05$ \\
& $3.00 \mathrm{~cd} / \mathrm{m}^{2}$ vs. $5.50 \mathrm{~cd} / \mathrm{m}^{2}$ & $p<.05$ \\
& $3.00 \mathrm{~cd} / \mathrm{m}^{2}$ vs. $8.00 \mathrm{~cd} / \mathrm{m}^{2}$ & $n . s$ \\
& $5.50 \mathrm{~cd} / \mathrm{m}^{2}$ vs. $8.00 \mathrm{~cd} / \mathrm{m}^{2}$ & $n . s$ \\
$6 \pi \mathrm{rad} / \mathrm{s}$ & $0.50 \mathrm{~cd} / \mathrm{m}^{2}$ vs. $3.00 \mathrm{~cd} / \mathrm{m}^{2}$ & $p<.05$ \\
& $0.50 \mathrm{~cd} / \mathrm{m}^{2}$ vs. $5.50 \mathrm{~cd} / \mathrm{m}^{2}$ & $p<.05$ \\
& $0.50 \mathrm{~cd} / \mathrm{m}^{2}$ vs. $8.00 \mathrm{~cd} / \mathrm{m}^{2}$ & $p<.05$ \\
& $3.00 \mathrm{~cd} / \mathrm{m}^{2}$ vs. $5.50 \mathrm{~cd} / \mathrm{m}^{2}$ & $n . s$ \\
& $3.00 \mathrm{~cd} / \mathrm{m}^{2}$ vs. $8.00 \mathrm{~cd} / \mathrm{m}^{2}$ & $p<.05$ \\
& $5.50 \mathrm{~cd} / \mathrm{m}^{2}$ vs. $8.00 \mathrm{~cd} / \mathrm{m}^{2}$ & $n . s$ \\
& $0.50 \mathrm{~cd} / \mathrm{m}^{2}$ vs. $3.00 \mathrm{~cd} / \mathrm{m}^{2}$ & $p<.05$ \\
& $0.50 \mathrm{~cd} / \mathrm{m}^{2}$ vs. $5.50 \mathrm{~cd} / \mathrm{m}^{2}$ & $p<.05$ \\
& $0.50 \mathrm{~cd} / \mathrm{m}^{2}$ vs. $8.00 \mathrm{~cd} / \mathrm{m}^{2}$ & $p<.05$ \\
& $3.00 \mathrm{~cd} / \mathrm{m}^{2}$ vs. $5.50 \mathrm{~cd} / \mathrm{m}^{2}$ & $n . s$ \\
& $3.00 \mathrm{~cd} / \mathrm{m}^{2}$ vs. $8.00 \mathrm{~cd} / \mathrm{m}^{2}$ & $p<.05$ \\
& $5.50 \mathrm{~cd} / \mathrm{m}^{2}$ vs. $8.00 \mathrm{~cd} / \mathrm{m}^{2}$ & $n . s$ \\
& $0.50 \mathrm{~cd} / \mathrm{m}^{2}$ vs. $3.00 \mathrm{~cd} / \mathrm{m}^{2}$ & $p<.05$ \\
& $0.50 \mathrm{~cd} / \mathrm{m}^{2}$ vs. $5.50 \mathrm{~cd} / \mathrm{m}^{2}$ & $p<.05$ \\
& $0.50 \mathrm{~cd} / \mathrm{m}^{2}$ vs. $8.00 \mathrm{~cd} / \mathrm{m}^{2}$ & $p<.05$ \\
& $3.00 \mathrm{~cd} / \mathrm{m}^{2}$ vs. $5.50 \mathrm{~cd} / \mathrm{m}^{2}$ & $n . s$ \\
& $3.00 \mathrm{~cd} / \mathrm{m}^{2}$ vs. $8.00 \mathrm{~cd} / \mathrm{m}^{2}$ & $p<.05$ \\
& $5.50 \mathrm{~cd} / \mathrm{m}^{2}$ vs. $8.00 \mathrm{~cd} / \mathrm{m}^{2}$ & $n . s$ \\
\hline & & \\
& &
\end{tabular}

\subsection{Discussion}

The results from Experiment 1 support the first working hypothesis that the edges' luminance contrasts determine the direction and amount of the fluttering-heart illusion. When the outer disk's edge had a high luminance contrast and the inner disk's edge had a low luminance contrast, i.e., under the 0.50 - and $3.00-\mathrm{cd} / \mathrm{m}^{2}$ conditions, the fluttering-heart illusion occurred even when the stimuli were achromatic. The direction of the fluttering-heart illusion was reversed when the outer disk's edge had a low luminance contrast and the inner disk's edge had a high luminance contrast, i.e., under the chromatic conditions of $8.00 \mathrm{~cd} / \mathrm{m}^{2}$. The amount of illusion decreased when the luminance contrasts of the outer and inner disks' edges were similar, i.e., under the chromatic and achromatic conditions of $3.00 \mathrm{~cd} / \mathrm{m}^{2}$. Thus, under these conditions, the luminance contrasts of the outer and inner disks' edges determined the direction and amount of the fluttering-heart illusion.

Although the results under the achromatic conditions of 5.50 and $8.00 \mathrm{~cd} / \mathrm{m}^{2}$ seem inexplicable in terms of the effect of the luminance contrasts of the outer and inner disks' edges, they are explicable by observing the interaction with mean luminance (Fig. 5). Under these conditions, the outer disk's edge had a low luminance contrast, and the inner disk's edge had a high luminance contrast. However, the mean luminance of the outer disk's edge, i.e., the background and outer disk's mean luminance, was high, and the mean luminance of the inner disk's edge, i.e., the outer and inner disks' mean luminance, was low. Therefore, the edge with a low luminance contrast and high mean luminance was quickly detected compared to the edge with a high luminance contrast and low mean luminance because brighter stimuli are detected faster compared to darker stimuli [24-27]. In future, we intend to examine this explanation further.

Differences between the chromatic and achromatic conditions indicate that color information processing contributes to the mechanism of the fluttering-heart illusion. Note that, in terms of color information processing, the fluttering-heart illusion under the achromatic condition is inexplicable, and this seems to indicate that the mechanism is independent of color information processing. However, one could not explain the results under the chromatic condition, especially differences from the results under the achromatic condition, without the contribution of color information processing. Thus, although it is not the primary mechanism, color information processing contributes to the mechanism causing the fluttering-heart illusion.

The results that the amount of the fluttering-heart illusion depended on the velocity of the disks' movements support the edge detection hypothesis. Figure 6 shows the explanation based on the edge detection hypothesis. In Fig. 6, the outer disk's edge has a high luminance contrast, and the inner disk's edge has a low luminance contrast. Movements are slow in Fig. 6a and fast in Fig. 6b. The slower latencies of the low contrast edges compared with the high contrast edges are the same in Fig. 6a, b. Note that the difference between the actual and perceived positions of the inner disk's edge is greater in Fig. 6b compared to that in Fig. 6a. That is, the amount of the fluttering-heart illusion increases with the increased velocity of the disks' movements. Thus, the mechanism that the amount of the fluttering-heart illusion depends on the velocity of the disks' movements is explained by the edge detection hypothesis.

\section{Experiment 2: effect of first- and second-order edges}

\subsection{Purpose}

We examine the effect of the first- and second-order edges on the fluttering-heart illusion to evaluate the second working hypothesis: the fluttering-heart illusion occurs when first- and second-order edges coexist. In this experiment, 


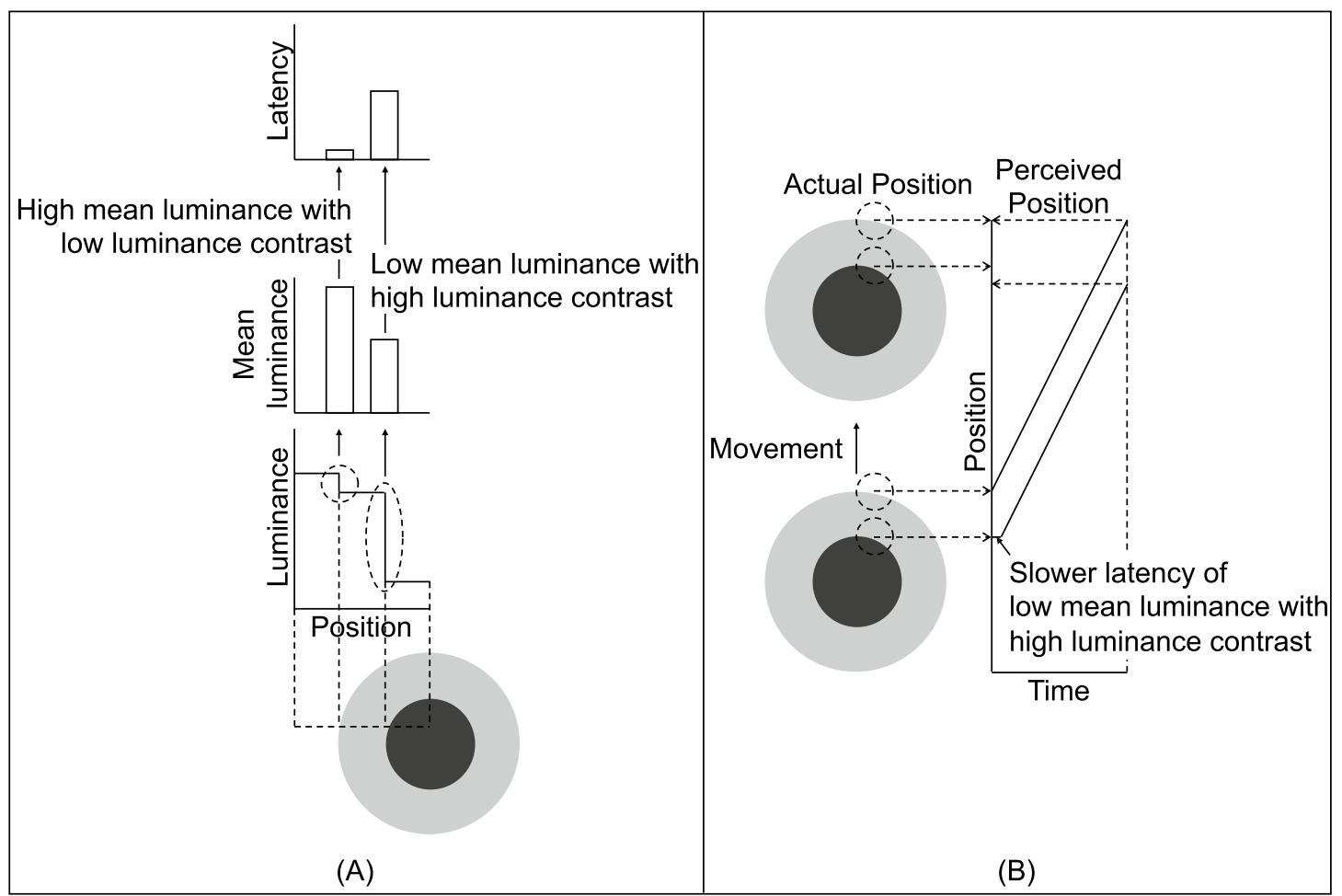

Fig. 5 Illustration showing the interaction between luminance contrast and mean luminance. a The low mean luminance with the high luminance contrast has a slower latency than the high mean luminance with the low luminance contrast. b The slower latency of the

the stimuli's background have a high luminance, the outer figures have a temporally modulated luminance, and the inner figures have a low luminance (Fig. 7). Under conditions in which the outer figures' mean luminance is the same as the background's luminance, the outer figures have second-order edges and the inner figures have first-order edges (Fig. 7a). Under conditions in which the outer figures' mean luminance is the same as the inner figures' luminance, the outer figures have first-order edges and the inner figures have second-order edges (Fig. 7b). We measure the direction and amount of the fluttering-heart illusion under these conditions.

\subsection{Method}

The stimuli and observers in Experiment 2 differed from those in Experiment 1, but the other methods in Experiment 2 were the same as those in Experiment 1. The details of the stimuli and observers are as follows.

Figure 7 shows a schematic of the stimuli used in Experiment 2. The background was the same as in Experiment 1. The outer and inner disks had the same sizes and movements as in Experiment 1. The outer disk comprised dots, and each dot was $3 \mathrm{~min}$ arc wide and $3 \mathrm{~min}$ arc high. The outer disk low mean luminance with high luminance contrast causes the delay of the perceived position against the actual position when the figure moves

had a temporally modulated luminance. Each dot's luminance was 3.00 or $8.00 \mathrm{~cd} / \mathrm{m}^{2}$ under the $5.50-\mathrm{cd} / \mathrm{m}^{2}$ condition of the outer disk's mean luminance, 4.50 or $9.50 \mathrm{~cd} /$ $\mathrm{m}^{2}$ under the $7.00-\mathrm{cd} / \mathrm{m}^{2}$ condition of the outer disk's mean luminance, 6.00 or $11.0 \mathrm{~cd} / \mathrm{m}^{2}$ under the $8.50-\mathrm{cd} / \mathrm{m}^{2}$ condition of the outer disk's mean luminance, or 7.50 or $12.5 \mathrm{~cd} /$ $\mathrm{m}^{2}$ under the $10.0-\mathrm{cd} / \mathrm{m}^{2}$ condition of the outer disk's mean luminance, and was randomly determined with $50 \%$ probability every $16 \mathrm{~ms}$. The outer disk's mean luminance of $10.0 \mathrm{~cd} / \mathrm{m}^{2}$ was the same luminance as the background; therefore, the outer disk had the second-order edge under this condition (Fig. 7a). The outer disk's mean luminance of $5.50 \mathrm{~cd} / \mathrm{m}^{2}$ was the same luminance as the inner disk that had a homogeneous luminance of $5.50 \mathrm{~cd} / \mathrm{m}^{2}$; therefore, the inner disk had the second-order edge under this condition (Fig. 7b). Thus, the outer disk had the first-order edge and the inner disk had the second-order edge under the 5.50-cd/ $\mathrm{m}^{2}$ condition of the outer disk's mean luminance, and the outer disk had the second-order edge and the inner disk had the first-order edge under the $10.0-\mathrm{cd} / \mathrm{m}^{2}$ condition of the outer disk's mean luminance.

One employee who had participated in Experiment 1 and six undergraduate students from Tokiwa University participated in Experiment 2. One of them was the author and 


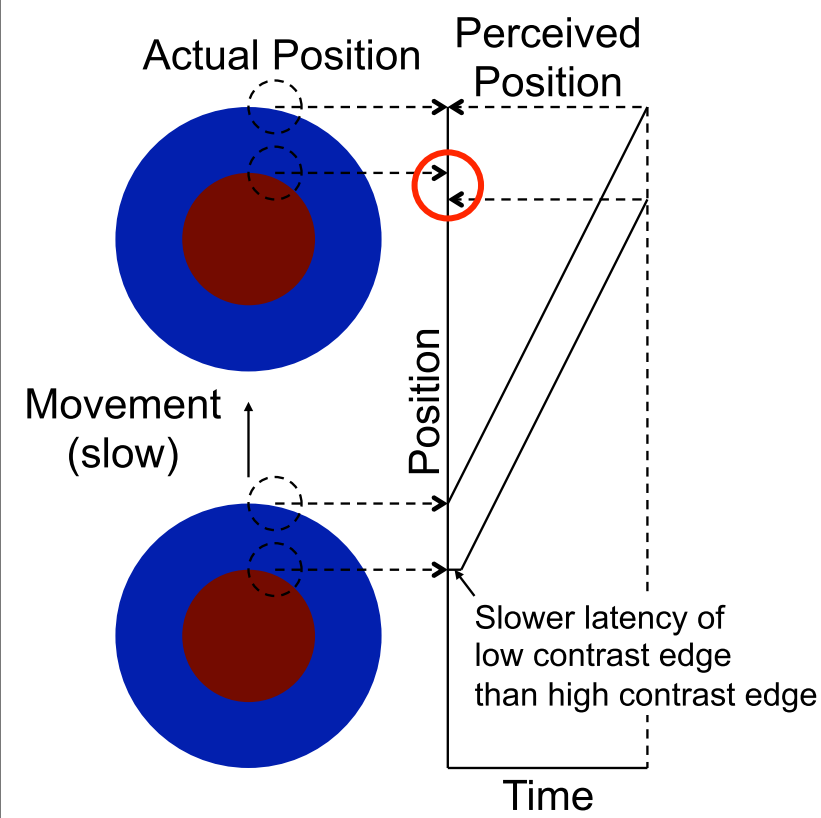

(A)

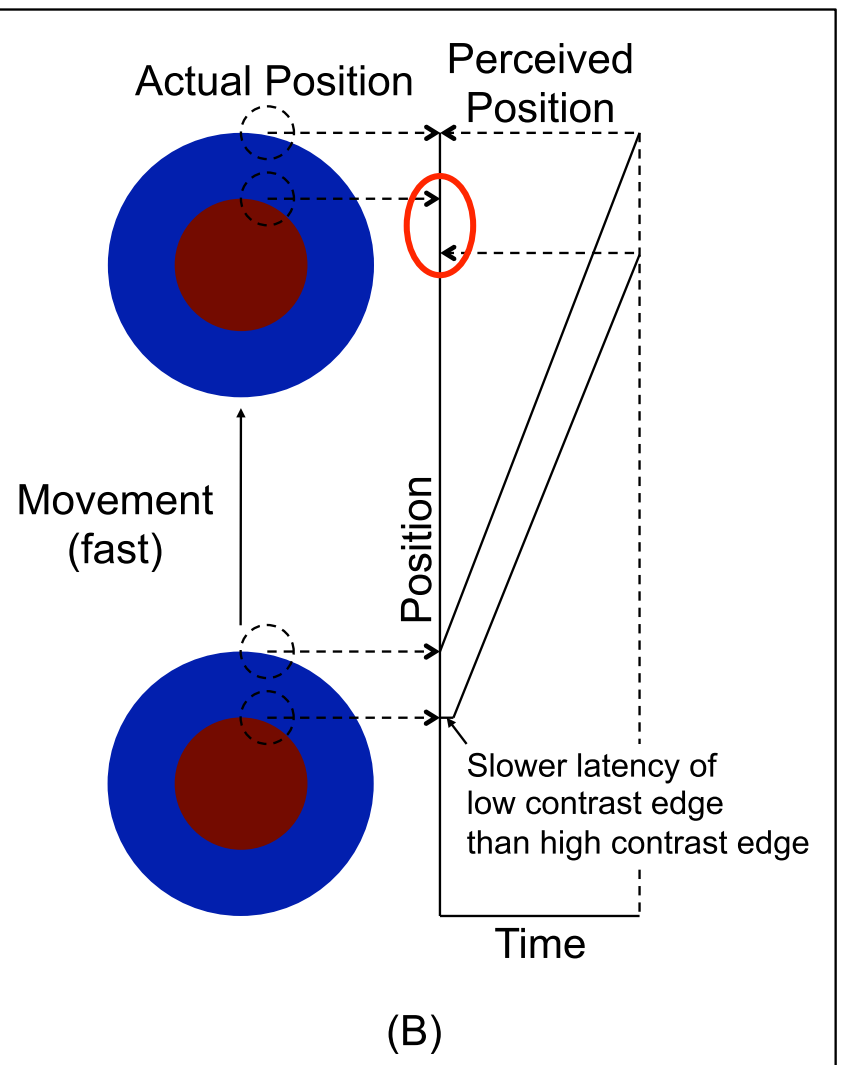

Fig. 6 Illustration showing the explanation of the effect of the velocity of disks' movements. a Case where movement is slow. b Case where movement is fast

the rest were naive to the purpose of the experiment. All observers had normal or corrected-to-normal visual acuity and normal color vision.

\subsection{Results}

Each observer's mean PSE was first calculated based on 10 trials for each condition, and the seven observers' mean PSE and $95 \%$ confidence interval were then calculated according to each observer's mean PSE for each condition. Figure 8 shows the seven observers' mean PSE plus/minus 95\% confidence interval as a function of the outer disk's mean luminance. The $95 \%$ confidence interval did not include $0 \pi$ rad of PSE under $5.50 \mathrm{~cd} / \mathrm{m}^{2}$ of all the velocity conditions, and the PSE had a positive value. These results indicate that the inner disk's movement appears delayed against the outer disk's movement. The $95 \%$ confidence interval did not include $0 \pi \mathrm{rad}$ of PSE under $10.0 \mathrm{~cd} / \mathrm{m}^{2}$ of all the velocity conditions, and the PSE had a negative value. These results indicate that the outer disk's movement appears delayed against the inner disk's movement. Thus, the fluttering-heart illusion occurs under these conditions, but the direction of illusion is reversed under $10.0 \mathrm{~cd} / \mathrm{m}^{2}$ of all the velocity conditions.
A two-way (outer disk's mean luminance $\times$ velocity of disks' movements) ANOVA was conducted because Mauchly's sphericity test did not reject the sphericity assumption. Tables 5 and 6 show the results of those statistical tests. The interaction between the outer disk's mean luminance and the velocity of the disks' movements was significant. The effect of the outer disk's mean luminance was significant. The effect of the velocity of the disk's movements was insignificant. These results indicate that the effect of the outer disk's mean luminance interacts with the velocity of the disks' movements.

Multiple comparisons using Bonferroni's method were conducted to further examine the interaction between the outer disk's mean luminance and the velocity of the disks' movements. Table 7 shows the results of those statistical tests. Under the condition of $4 \pi \mathrm{rad} / \mathrm{s}$, the pair in the 5.50and $8.50-\mathrm{cd} / \mathrm{m}^{2}$ conditions, the pair in the $5.50-$ and $10.0-\mathrm{cd} /$ $\mathrm{m}^{2}$ conditions, the pair in the $7.00-$ and $10.0-\mathrm{cd} / \mathrm{m}^{2}$ conditions, and the pair in the $8.50-$ and $10.0-\mathrm{cd} / \mathrm{m}^{2}$ conditions had significant differences. Under the conditions of $6 \pi, 8 \pi$, and $10 \pi \mathrm{rad} / \mathrm{s}$, except for the pair in the $7.00-$ and $8.50-\mathrm{cd} /$ $\mathrm{m}^{2}$ conditions, all pairs of the outer disk's mean luminance had significant differences. These results indicated that the luminance contrasts of the outer and inner disks' edges affect 


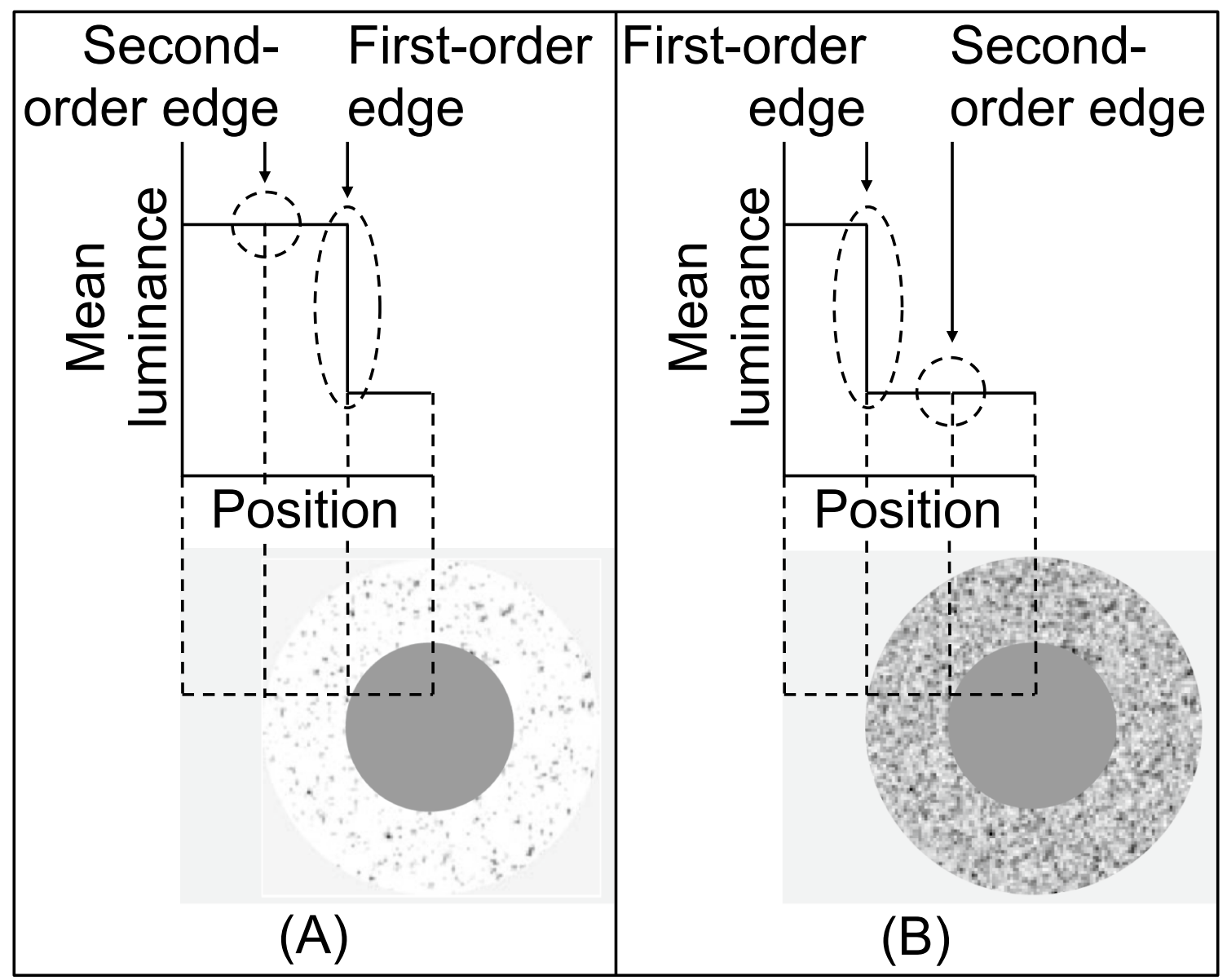

Fig. 7 Schematic of stimuli used in Experiment 2. a Condition where the outer disk's mean luminance is the same as the background's luminance. b Condition where the outer disk's mean luminance is the same as the inner disk's luminance

the fluttering-heart illusion and such effects depend on the velocity of the disks' movements.

\subsection{Discussion}

The results that the fluttering-heart illusion occurred under the $5.50 \mathrm{~cm} / \mathrm{m}^{2}$ conditions support the second working hypothesis. Under these conditions, the outer disk had the first-order edge, and the inner disk had the second-order edge. That is, the latency of detecting the inner disk's edge was slower than the latency of detecting the outer disk's edge. This caused the inner disk's movement that appeared delayed against the outer disk's movement. Thus, the fluttering-heart illusion occurred when the first- and second-order edges coexisted.

The results that the fluttering-heart illusion was reversed under the $10.0-\mathrm{cd} / \mathrm{m}^{2}$ conditions also support the second working hypothesis. Under these conditions, the outer disk had the second-order edge, and the inner disk had the firstorder edge. That is, the latency of detecting the outer disk's edge was slower than the latency of detecting the inner disk's edge. This caused the outer disk's movement that appeared delayed against the inner disk's movement. Thus, the fluttering-heart illusion was reversed even when the firstand second-order edges coexisted.

We need to further examine the fluttering-heart illusion under conditions in which the first- and second-order edges coexist. In Experiment 2, second-order edges were defined by a temporal modulation in luminance. However, features that can define second-order edges are not only temporal modulation in luminance. For example, differences in texture or color can define second-order edges [18-23]. Examination under conditions in which second-order edges are defined by such features is necessary, and this is our future work.

The results that the amount of the fluttering-heart illusion depended on the velocity of the disks' movements support the edge detection hypothesis. The explanation in the last paragraph of Sect. 2.3 can apply to conditions where the first- and second-order edges coexist (Fig. 9). In Fig. 9, outer disks have first-order edges, and inner disks have second-order edges. Movements are slow in Fig. 9a and fast in 


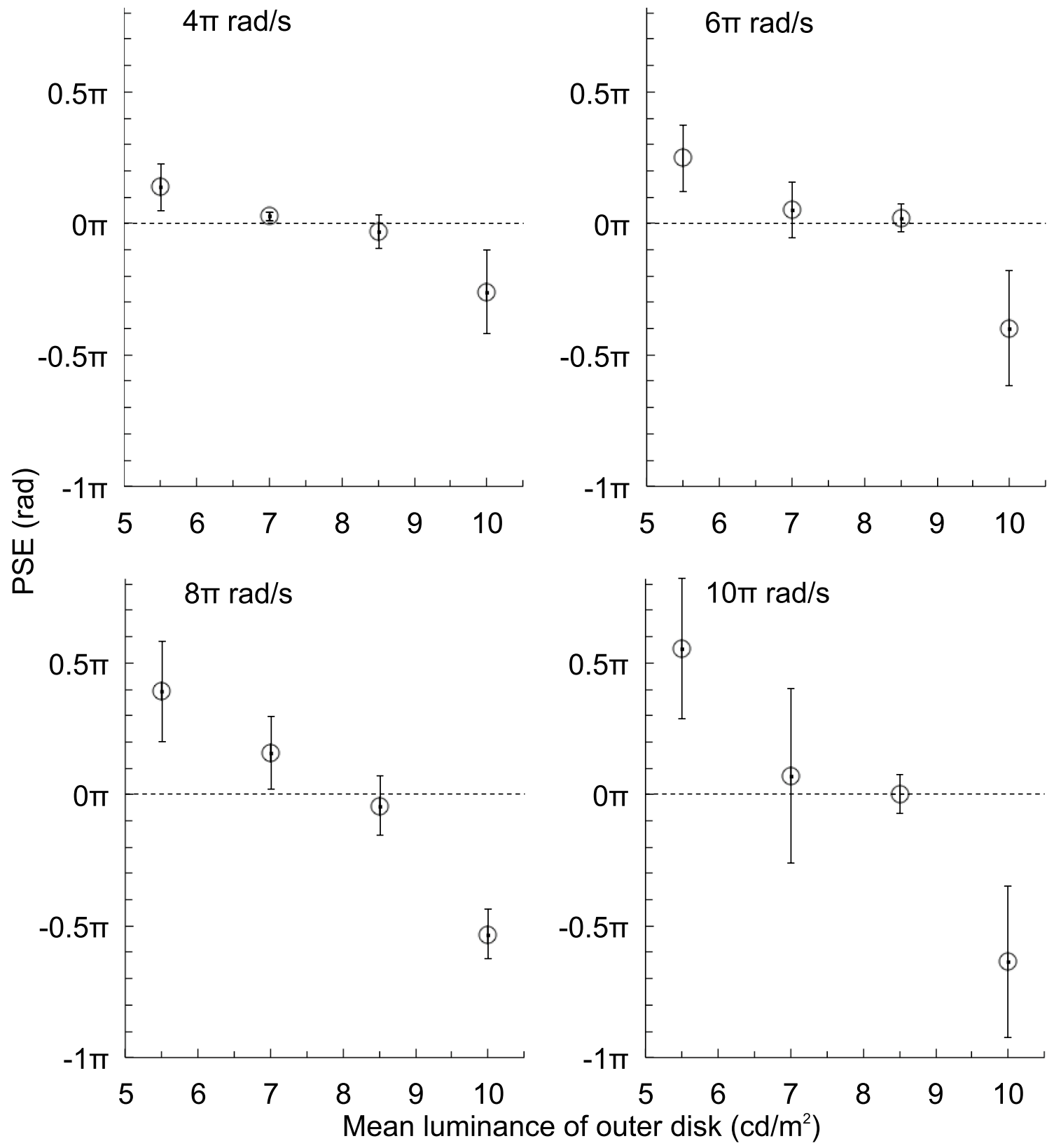

Fig. 8 Seven observers' mean PSE plus/minus 95\% confidence interval as a function of the outer disk's mean luminance. The positive or negative PSE indicates the same as Fig. 7. The error bar represents the $95 \%$ confidence interval

Table 5 Results of the Mauchly's sphericity test in Experiment 2

\begin{tabular}{lllll}
\hline Factor & $W$ & $\chi^{2}$ & $\mathrm{~d} f$ & $p$ \\
\hline Outer disk's mean luminance & .100 & 10.9 & 5 & .058 \\
Velocity of disks' movements & .210 & 7.38 & 5 & .202 \\
\hline
\end{tabular}

Fig. 9b. The slower latencies of the second-order edges compared with first-order edges are the same in Fig. 9a, b. Note that the difference between the actual and perceived positions of the inner disk's edge is greater in Fig. 9a compared
Table 6 Results of the two-way ANOVA in Experiment 2

\begin{tabular}{lcllll}
\hline Factor & $F$ & $d f$ & $M S E$ & $p$ & $\eta_{\mathrm{p}}{ }^{2}$ \\
\hline $\begin{array}{l}\text { Outer disk's mean luminance } \\
\text { Velocity of disks' movements }\end{array}$ & 9.37 & 9,54 & 0.02 & .000 & .610 \\
Outer disk's mean luminance & 81.3 & 3,18 & 0.05 & .000 & .931 \\
Velocity of disks' movements & 0.39 & 3,18 & 0.02 & .759 & .061 \\
\hline
\end{tabular}


Table 7 Results of the multiple comparisons to examine the interaction between the outer disk's mean luminance and the velocity of the disks' movements in Experiment 2

\begin{tabular}{|c|c|c|}
\hline $\begin{array}{l}\text { Velocity of disks' } \\
\text { movements }\end{array}$ & Outer disk's mean luminance & Significance \\
\hline \multirow[t]{6}{*}{$4 \pi \mathrm{rad} / \mathrm{s}$} & $5.50 \mathrm{~cd} / \mathrm{m}^{2}$ vs. $7.00 \mathrm{~cd} / \mathrm{m}^{2}$ & $n . s$ \\
\hline & $5.50 \mathrm{~cd} / \mathrm{m}^{2}$ vs. $8.50 \mathrm{~cd} / \mathrm{m}^{2}$ & $p<.05$ \\
\hline & $5.50 \mathrm{~cd} / \mathrm{m}^{2}$ vs. $10.0 \mathrm{~cd} / \mathrm{m}^{2}$ & $p<.05$ \\
\hline & $7.00 \mathrm{~cd} / \mathrm{m}^{2}$ vs. $8.50 \mathrm{~cd} / \mathrm{m}^{2}$ & $n . s$ \\
\hline & $7.00 \mathrm{~cd} / \mathrm{m}^{2}$ vs. $10.0 \mathrm{~cd} / \mathrm{m}^{2}$ & $p<.05$ \\
\hline & $8.50 \mathrm{~cd} / \mathrm{m}^{2}$ vs. $10.0 \mathrm{~cd} / \mathrm{m}^{2}$ & $p<.05$ \\
\hline \multirow[t]{6}{*}{$6 \pi \mathrm{rad} / \mathrm{s}$} & $5.50 \mathrm{~cd} / \mathrm{m}^{2}$ vs. $7.00 \mathrm{~cd} / \mathrm{m}^{2}$ & $p<.05$ \\
\hline & $5.50 \mathrm{~cd} / \mathrm{m}^{2}$ vs. $8.50 \mathrm{~cd} / \mathrm{m}^{2}$ & $p<.05$ \\
\hline & $5.50 \mathrm{~cd} / \mathrm{m}^{2}$ vs. $10.0 \mathrm{~cd} / \mathrm{m}^{2}$ & $p<.05$ \\
\hline & $7.00 \mathrm{~cd} / \mathrm{m}^{2}$ vs. $8.50 \mathrm{~cd} / \mathrm{m}^{2}$ & $n . s$ \\
\hline & $7.00 \mathrm{~cd} / \mathrm{m}^{2}$ vs. $10.0 \mathrm{~cd} / \mathrm{m}^{2}$ & $p<.05$ \\
\hline & $8.50 \mathrm{~cd} / \mathrm{m}^{2}$ vs. $10.0 \mathrm{~cd} / \mathrm{m}^{2}$ & $p<.05$ \\
\hline \multirow[t]{6}{*}{$8 \pi \mathrm{rad} / \mathrm{s}$} & $5.50 \mathrm{~cd} / \mathrm{m}^{2}$ vs. $7.00 \mathrm{~cd} / \mathrm{m}^{2}$ & $p<.05$ \\
\hline & $5.50 \mathrm{~cd} / \mathrm{m}^{2}$ vs. $8.50 \mathrm{~cd} / \mathrm{m}^{2}$ & $p<.05$ \\
\hline & $5.50 \mathrm{~cd} / \mathrm{m}^{2}$ vs. $10.0 \mathrm{~cd} / \mathrm{m}^{2}$ & $p<.05$ \\
\hline & $7.00 \mathrm{~cd} / \mathrm{m}^{2}$ vs. $8.50 \mathrm{~cd} / \mathrm{m}^{2}$ & $n . s$ \\
\hline & $7.00 \mathrm{~cd} / \mathrm{m}^{2}$ vs. $10.0 \mathrm{~cd} / \mathrm{m}^{2}$ & $p<.05$ \\
\hline & $8.50 \mathrm{~cd} / \mathrm{m}^{2}$ vs. $10.0 \mathrm{~cd} / \mathrm{m}^{2}$ & $p<.05$ \\
\hline \multirow[t]{6}{*}{$10 \pi \mathrm{rad} / \mathrm{s}$} & $5.50 \mathrm{~cd} / \mathrm{m}^{2}$ vs. $7.00 \mathrm{~cd} / \mathrm{m}^{2}$ & $p<.05$ \\
\hline & $5.50 \mathrm{~cd} / \mathrm{m}^{2}$ vs. $8.50 \mathrm{~cd} / \mathrm{m}^{2}$ & $p<.05$ \\
\hline & $5.50 \mathrm{~cd} / \mathrm{m}^{2}$ vs. $10.0 \mathrm{~cd} / \mathrm{m}^{2}$ & $p<.05$ \\
\hline & $7.00 \mathrm{~cd} / \mathrm{m}^{2}$ vs. $8.50 \mathrm{~cd} / \mathrm{m}^{2}$ & $n . s$ \\
\hline & $7.00 \mathrm{~cd} / \mathrm{m}^{2}$ vs. $10.0 \mathrm{~cd} / \mathrm{m}^{2}$ & $p<.05$ \\
\hline & $8.50 \mathrm{~cd} / \mathrm{m}^{2}$ vs. $10.0 \mathrm{~cd} / \mathrm{m}^{2}$ & $p<.05$ \\
\hline
\end{tabular}

to that in Fig. 9a. That is, the amount of the fluttering-heart illusion increases with the increased velocity of the disks' movements. Thus, the edge detection hypothesis can explain that the amount of the fluttering-heart illusion depends on the velocity of the disks' movements when first- and secondorder edges coexist.

The amount of the fluttering-heart illusion in Experiment 2 was larger than that in Experiment 1, and this is explicable based on the edge detection hypothesis. In Experiment 1, the slower latency of detecting the low luminance contrast edge than the latency of detecting the high luminance contrast edge caused the illusion. Note that both low luminance contrast edge and high luminance contrast edge were the firstorder edges. That is, the first-order edge detection mechanism detected them. In Experiment 2, the slower latency of detecting the second-order edge compared with the latency of detecting the first-order edge caused the illusion. Note that detecting the second-order edge was after detecting the first-order edge. That is, the differences in the latencies of edge detection were larger in Experiment 2 than in Experiment 1, and this caused the larger amount of the illusion in Experiment 2 than in Experiment 1 (see Fig. 2). Thus, the edge detection hypothesis can explain the results that the amount of the illusion became larger in Experiment 2 than in Experiment 1.

The variance of the amount of the fluttering-heart illusion in Experiment 2 was also larger than that in Experiment 1, and this is explicable based on individual differences in the perception of luminance. In Experiment 2, we defined the second-order edge as the outer disk's edge under the conditions where the background and the outer disk had equiluminance, or as the inner disk's edge under the conditions where the outer disk and the inner disk had equiluminance. These equiluminances were not based on each observer's perception of luminance. The observers who perceived equiluminance and the second-order edges had a large amount of the illusion, whereas the observers who did not perceive equiluminance and perceived the first-order edges had a small amount of the illusion. Such differences between the observers caused the larger variance of the amount of the illusion in Experiment 2 than in Experiment 1. We intend to examine by using equiluminance based on each observer's perception of luminance in the near future.

\section{General discussion}

As described in Sects. 2 and 3, the results obtained from Experiments 1 and 2 supported the first and second working hypotheses, respectively; therefore, these results support the edge detection hypothesis. Edges' luminance contrasts determined the direction and amount of the fluttering-heart illusion in Experiment 1. Differences in latencies of detection between the first- and second-order edges caused the fluttering-heart illusion in Experiment 2. These results and a series of phenomena reported in previous studies [1-10] were explicable by the edge detection hypothesis. Thus, we can conclude that the edge detection hypothesis is a valid hypothesis for explaining the mechanism causing the fluttering-heart illusion.

Note that supporting the edge detection hypothesis does not controvert other hypotheses on the mechanism causing the fluttering-heart illusion. For example, color information processing contributed to the mechanism causing the fluttering-heart illusion in Experiment 1, although it was not the main mechanism (see the eighth paragraph in Sect. 2.3). The fluttering-heart illusion would occur based on only color information processing if the luminance contrasts of the outer and inner figures' edges were the same. Thus, other hypotheses should examine them under conditions where the luminance contrasts of the outer and inner figures' edges are the same, and this is our future work.

Additionally, we need to further examine the edge detection hypothesis to display the mechanism causing the fluttering-heart illusion. The interaction between the edges' 


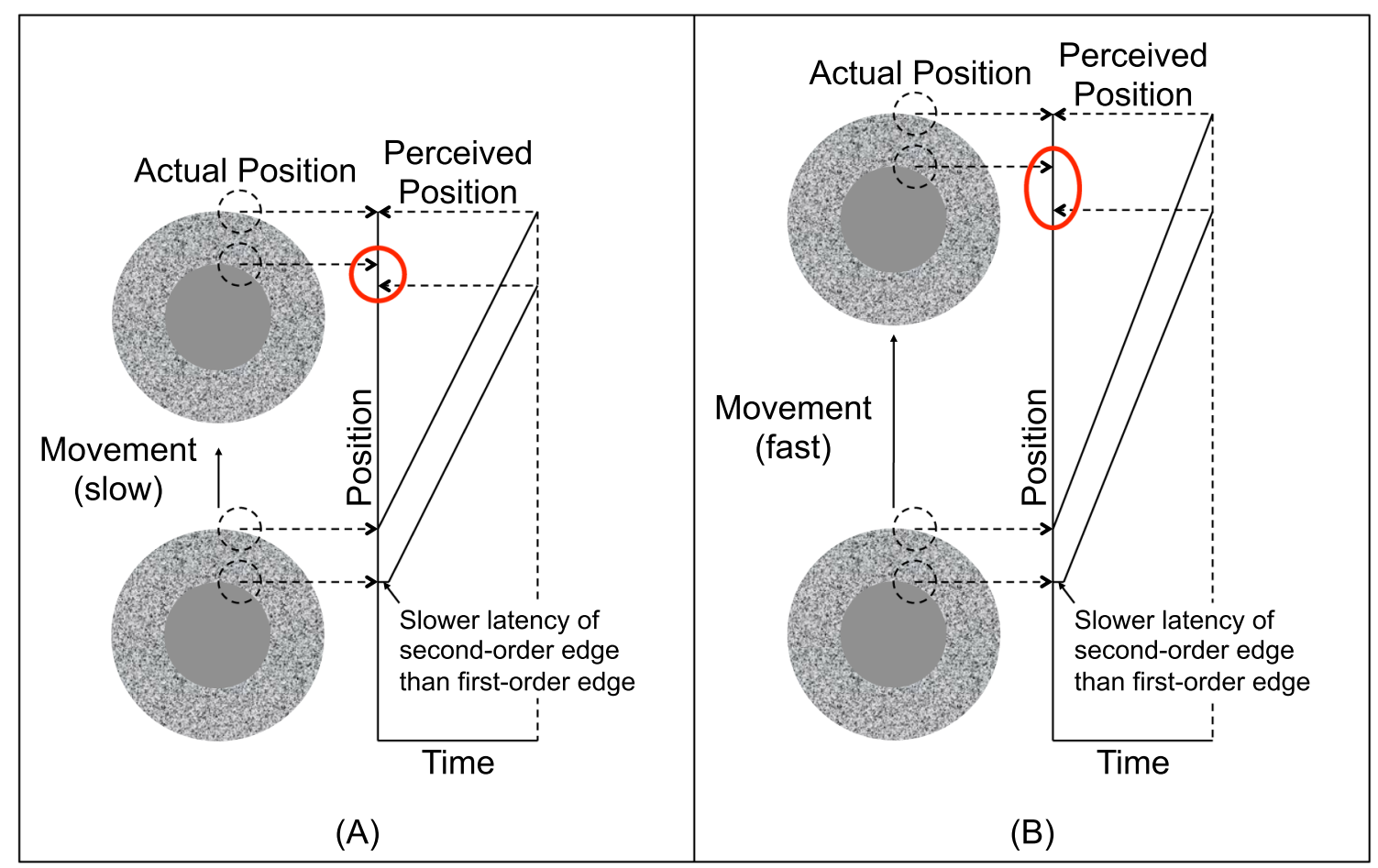

Fig. 9 Illustration presenting the explanation of the effect of the velocity of disks' movements. a Case where movement is slow. b Case where movement is fast

luminance contrasts and the edges' mean luminance was unclear in Experiment 1 (see the sixth and seventh paragraphs in Sect. 2.3). Examination under conditions where the second-order edges were defined by differences in luminance or color was necessary for Experiment 2 (see the fifth paragraph in Sect. 3.3). Furthermore, we must examine other working hypotheses to evaluate the edge detection hypothesis. We intend to conduct such examinations in the near future.

\subsection{Fluttering-heart illusion in mesopic and photopic vision}

Some studies have reported that the fluttering-heart illusion more easily occurs in mesopic than in photopic vision [1-3]. For example, the fluttering-heart illusion occurs in dim light, but not in bright light when the same stimulus is observed. Such findings suggest that the mechanism causing the fluttering-heart illusion is strongly related to mesopic vision.

However, several studies have reported that the flutteringheart illusion also occurs in photopic vision $[9,10]$, and the reason for this inconsistency has yet to be clarified. In fact, this study's experiments were conducted in photopic vision, and the results indicated that the fluttering-heart illusion occurred. Clearly, photopic vision is also related to the mechanism causing the fluttering-heart illusion. We need to explain why the fluttering-heart illusion did not occur in photopic vision in some studies [1-3].

The edge detection hypothesis could explain this inconsistency on the basis of light reflectance. In studies which reported that the fluttering-heart illusion occurred only in mesopic vision, objects that reflected light but did not emit light were used for stimuli [1-3]. For instance, a piece of white paper is used for the stimulus, and the outer and inner figures are painted on the paper. The light reflectance of the paper, i.e. the background, is $80.0 \%$, that of the outer figure is $35.0 \%$, and that of the inner figure is $25.0 \%$. When the light source under which the luminance of a perfect reflecting diffuser (100\% reflecting white) is $4.00 \mathrm{~cd} / \mathrm{m}^{2}$ illuminates the background and figures, the luminance of the background is $3.20 \mathrm{~cd} / \mathrm{m}^{2}$, and the luminances of the outer and inner figures are 1.40 and $1.00 \mathrm{~cd} / \mathrm{m}^{2}$, respectively. Note that this luminance condition accords with mesopic vision. Although the outer edge's luminance contrast is not high, the inner edge's luminance contrast is very low. The latency of detection of the inner edge is very slow-slower than the latency of detection of the outer edge. Therefore, the fluttering-heart illusion occurs in mesopic vision. When the light source under which the luminance of a perfect reflecting diffuser (100\% reflecting white) is $100 \mathrm{~cd} / \mathrm{m}^{2}$ illuminates the background and figures, the luminance of the background is $80.0 \mathrm{~cd} / \mathrm{m}^{2}$, and the luminances of the outer and inner figures are 35.0 and $25.0 \mathrm{~cd} / \mathrm{m}^{2}$, respectively. Note that the 
luminance condition accords with photopic vision. Although the inner edge's luminance contrast is lower than the outer edge's luminance contrast, it is sufficiently high to detect the inner edge as fast as to detect the outer edge. Therefore, the fluttering-heart illusion does not occur in photopic vision. Thus, the edge detection hypothesis could explain the reason for the inconsistency, and we intend to examine this explanation in the near future.

\subsection{Edge detection hypothesis and motion detection mechanism}

Although the edge detection hypothesis is valid for explaining the mechanism causing the fluttering-heart illusion, its relation to the motion detection mechanism is not clear. In the edge detection hypothesis, the mechanism causing the fluttering-heart illusion is explained without the motion detection mechanism (see Fig. 2). However, the flutteringheart illusion is a phenomenon of motion perception; therefore, the mechanism causing it is obviously related to the motion detection mechanism. We need to relate the edge detection hypothesis to motion detection mechanism.

The effect of luminance contrast on motion perception would be important for clarifying the relation between the edge detection hypothesis and the motion detection mechanism. In particular, the effect on the perception of global motion would be crucial because the perceived motion in the fluttering-heart illusion is global motion. According to previous studies, the increment of luminance contrast facilitates the perception of global motion [28]. In other words, the perception of global motion is made difficult with decreasing luminance contrast. Such phenomena are explained based on the characteristics of the motion detection mechanism [28]. That is, when luminance contrast is low, local motion detectors generate weak motion signals, and these weak motion signals are input into global motion detectors. As the results, the perception of global motion is made difficult. We could relate the edge detection hypothesis to the motion detection mechanism if the slow latencies of edge detection were relevant to generating weak motion signals. This is our future work.

\subsection{Effects of physical afterimages caused by characteristics of liquid crystal displays (LCDs)}

Although no observers reported any afterimages of the stimuli in Experiments 1 and 2, there were physical afterimages caused by the characteristics of LCDs. According to previous studies, actual exposure duration of stimuli presented with LCDs becomes longer than exposure duration calculated from the refresh rate of video cards [29]. Response time of LCDs causes such phenomena. When the actual exposure duration of one video frame is longer than the exposure duration calculated from the refresh rate, motion stimuli are with physical afterimages. Thus, we need to discuss the effects of such physical afterimages on the results obtained from Experiments 1 and 2.

The results obtained from Experiments 1 and 2 would be inexplainable based on physical afterimages caused by the characteristics of LCDs. To explain the results of Experiment 1, we need assuming that the effect of physical afterimages on edge detection differs between conditions of luminance contrast. For instance, physical afterimages facilitate high luminance contrast edge detection and obstruct low luminance contrast edge detection. Similarly, the assumption that the effect of physical afterimages differs between the first- and second-order edges is necessary for explaining the results of Experiment 2. For instance, physical afterimages facilitate detecting the first-order edges and obstruct detecting the second-order edges. However, such assumptions seem to unreasonable because physical afterimages would work as noise in the edge detection mechanism. It seems reasonable to assume physical afterimages obstruct detecting any edges. This assumption means that the results of Experiments 1 and 2 are inexplainable based on physical afterimages. We intend to examine these assumptions in the near future.

\section{Conclusion}

This paper described two experiments examining the mechanism causing the fluttering-heart illusion. In Experiment 1 , the effect of edges' luminance contrasts on the fluttering-heart illusion was examined, and edges' luminance contrasts determined the direction and amount of the illusion. Experiment 2 examined the first- and secondorder edges' effect on the fluttering-heart illusion and differences in the latencies of detection between the first- and second-order edges were observed to cause the illusion. The results obtained from Experiments 1 and 2 indicated that differences in the latencies of edge detection caused the fluttering-heart illusion.

Acknowledgements This study is a revision of the authors' poster presentations at the 2014 winter meeting of the Vision Society of Japan, the 10th Asia-Pacific Conference on Vision, and the 37th European Conference on Visual Perception.

\section{Compliance with ethical standards}

Conflict of interest There are no conflicts of interest to declare.

Ethical approval This study was approved by the Ethical Review Board for the use of human subjects of Kanagawa Institute of Technology. 
Open Access This article is licensed under a Creative Commons Attribution 4.0 International License, which permits use, sharing, adaptation, distribution and reproduction in any medium or format, as long as you give appropriate credit to the original author(s) and the source, provide a link to the Creative Commons licence, and indicate if changes were made. The images or other third party material in this article are included in the article's Creative Commons licence, unless indicated otherwise in a credit line to the material. If material is not included in the article's Creative Commons licence and your intended use is not permitted by statutory regulation or exceeds the permitted use, you will need to obtain permission directly from the copyright holder. To view a copy of this licence, visit http://creativecommons.org/licenses/by/4.0/.

\section{References}

1. Brewster D (1844) On the same subject. Rep Br Ass Advmt Sci Trans Sect 10

2. Von Helmholtz, H.: Handbuch der physiologischen Optik. L. Voss, Leipzig, Germany (1867)

3. Wheatstone C (1844) On the singular effect of the juxtaposition of certain colours under particular circumstances. Rep Br Ass Advmt Sci Trans Sect 10

4. Von Kries, J.: Über die Wirkung kurzdauernder Lichtreize auf das Sehorgan. Z Psychol Physiol Sinnesorg 12, 81-101 (1896)

5. Von Grünau, M.W.: The "fluttering heart" and spatio-temporal characteristics of color processing-I: Reversibility and the influence of luminance. Vision Res. 15, 431-436 (1975)

6. Von Grünau, M.W.: The "fluttering heart" and spatio-temporal characteristics of color processing-II: Lateral interactions across the chromatic border. Vision Res 15, 437-440 (1975)

7. Von Grünau, M.W.: The "Fluttering Heart" and spatio-temporal characteristics of color processing-III: Interactions between the systems of the rods and the long-wavelength cones. Vision Res. 16, 397-401 (1976)

8. Anstis, S., Macleod, D.: Why hearts flutter: distorted dim motions. J. Vis. 15(3), 23 (2015)

9. Nguyen-Tri, D., Faubert, J.: The fluttering-heart illusion: A new hypothesis. Perception. 32, 627-634 (2003)

10. Kitaoka, A., Ashida, H.: A variant of the anomalous motion illusion based upon contrast and visual latency. Perception. 36, 1019-1035 (2007)

11. Benardete, E.A., Kaplan, E.: The dynamics of primate $M$ retinal ganglion cells. Vis Neurosci 16, 355-368 (1999)

12. Benardete, E.A., Kaplan, E., Knight, B.W.: Contrast gain control in the primate retina: $\mathrm{P}$ cells are not $\mathrm{X}$-like, some $\mathrm{M}$ cells are. Vis Neurosci 8, 483-486 (1992)

13. Shapley, R.M., Victor, J.D.: The effect of contrast on the transfer properties of cat retinal ganglion cells. J Physiol 285, 275-298 (1978)
14. Shapley, R.M., Victor, J.D.: Nonlinear spatial summation and the contrast gain control of cat retinal ganglion cells. J Physiol 290, 141-161 (1979)

15. Shapley, R.M., Victor, J.D.: How the contrast gain control modifies the frequency responses of cat retinal ganglion cells. J Physiol 318, 161-179 (1981)

16. Lee, B.B., Pokorny, J., Smith, V.C., Kremers, J.: Responses to pulses and sinusoids in macaque ganglion cells. Vision Res $\mathbf{3 4}$, 3081-3096 (1994)

17. Stromeyer, C.F., Martini, P.: Human temporal impulse response speeds up with increased stimulus contrast. Vision Res 43, 285298 (2003)

18. Graham, N., Beck, J., Sutter, A.: Nonlinear processes in spatialfrequency channel models of perceived texture segregation: effects of sign and amount of contrast. Vision Res 32, 719-743 (1992)

19. Grossberg, S., Mingolla, E.: Neural dynamics of perceptual grouping: textures, boundaries, and emergent segmentations. Percept Psychophys 38, 141-171 (1985)

20. Landy, M.S., Bergen, J.R.: Texture segregation and orientation gradient. Vision Res. 31, 679-691 (1991)

21. Sutter, A., Sperling, G., Chubb, C.: Measuring the spatial frequency selectivity of second-order texture mechanisms. Vision Res 35, 915-924 (1995)

22. Victor, J.D.: Models for preattentive texture discrimination: Fourier analysis and local feature processing in a unified framework. Spat Vis 3, 263-280 (1988)

23. Victor, J.D., Conte, M.M.: Cortical interactions in texture processing: scale and dynamics. Vis Neurosci 2, 297-313 (1989)

24. Baylor, D.A., Hodgkin, A.L.: Changes in time scale and sensitivity in turtle photoreceptors. J Physiol 242, 729-758 (1974)

25. Kelly, D.H.: Visual response to time-dependent stimuli I Amplitude sensitivity measurements. J Opt Soc Am 51, 422-429 (1961)

26. Kelly, D.H.: Theory of flicker and transient responses. I. Uniform fields. J. Opt Soc Am 61, 537-546 (1971)

27. Purpura, K., Tranchina, D., Kaplan, E., Shapley, R.M.: Light adaptation in the primate retina: analysis of changes in gain and dynamics of monkey retinal ganglion cells. Vis Neurosci 4, 75-93 (1990)

28. Edwards, M., Badcock, D.R., Nishida, S.: Contrast sensitivity of the motion system. Vision Res 36, 2411-2421 (1996)

29. Elze, T.: Misspecifications of stimulus presentation durations in experimental psychology: A systematic review of the psychophysics literature. PLoS ONE 5, e12792-1-e12792-7 (2010)

Publisher's Note Springer Nature remains neutral with regard to jurisdictional claims in published maps and institutional affiliations. 